Research Paper:

\title{
The Quality of Life of Patients With Different Leukemia Types
}

\author{
Hossein Poorcheraghi ${ }^{1}$ (D), Davood Hekmatpou ${ }^{2}$ (D) Fatemeh Mehrabi $^{* *}$ (i)
}

1. Student Research Committee, Arak University of Medical Sciences, Arak, Iran.

2. Department of Nursing, School of Nursing and Midwifery, Arak University of Medical Sciences, Arak, Iran.

\begin{tabular}{|l|l|}
\hline $\begin{array}{c}\text { use your devic to scan } \\
\text { and read the article online }\end{array}$ & $\begin{array}{l}\text { Cltation: Poorcheraghi, H., Hekmatpou, D., Mehrabi, M,. 2019. The Quality of Life of Patients With Different Leukemia } \\
\text { Types. Journal of Client-Centered Nursing Care, 5(2), pp. 97-104. https://doi.org/10.32598/JCCNC.5.2.97 }\end{array}$ \\
dol https://doi.org/10.32598/JCCNC.5.2.97
\end{tabular}

\section{(1) (\$)}

Article info:

Received: 18 Dec 2018

Accepted: 23 Mar 2019

Published: 01 May 2019
Keywords:

Quality of Life (QoL), Lymphocytic leukemia, Myeloid leukemia

\begin{abstract}
A B S T RA C T
Background: Leukemia is among the major causes of mortality and disability and the seventh most prevalent cancer worldwide. It has various effects on the biopsychosocial, reproductive, and economic performance of individuals. This study compared the Quality of Life (QoL) of hospitalized patients with different leukemia.
\end{abstract}

Methods: This was a descriptive cross-sectional study. In total, 100 adolescent patients with leukemia hospitalized in Ayatollah Khansari Hospital in Arak City, Iran, were selected by purposive sampling method. The samples were evaluated for six months in 2018. The required data were collected by EORTC-QLQ-C30 Quality of Life questionnaire and analyzed by descriptive statistics (Mean \pm SD and frequency) and Chi-squared test, Fisher's exact test, and $\mathrm{t}$-test in SPSS. $\mathrm{P} \leq 0.5$ was considered as significant.

Results: The overall QoL Mean \pm SD score was $62.2 \pm 12.59$, which was at a relatively appropriate level. The Mean \pm SD scores of life quality were $60.10 \pm 14.31$ for chronic lymphocytic leukemia, $59.51 \pm 12.83$ for acute lymphocytic leukemia, $66.61 \pm 10.94$ for chronic myeloid leukemia, and $62.84 \pm 6.33$ for acute myeloid leukemia. There were significant differences between the total QoL and its dimensions in different leukemia types $(\mathrm{P}<0.05)$. There was also a significant relationship between age, residency, and marital status of the patients, and their total QoL $(\mathrm{P}<0.05)$.

Conclusion: Patients with myeloid leukemia had a better QoL, compared to those with lymphocytic leukemia. The patients had better performance in the physical area and weaker performance in social domain. The knowledge of nurses and other healthcare members about these findings could be used to promote care and improve the QoL of patients with different leukemia types.

\section{* Corresponding Author:}




\section{Highlights}

- There was a significant difference between the total quality of life and its dimensions in different leukemia types.

- Patients with myeloid leukemia had a better QoL, compared to those with lymphocytic leukemia.

- Patients with leukemia had better performance in the physical area and weaker performance in the social domain.

- There was a significant relationship between the age, residency, and marital status of patients with leukemia and their total quality of life.

\section{Plain Language Summary}

The current study assessed the quality of life of patients with different leukemia types in a hospital in Arak City, Iran. Moreover, the total life quality of these patients was at a relatively appropriate level; although it differed in various leukemia types. Therefore, there is a need to consider the leukemia type while providing these patients with biosocial, emotional, and cognitive needs. Generally, these patients had better and poorer performance in physical and social domains, respectively, in terms of life quality. It means that more attention should be paid to the social needs of these patients.

\section{Background}

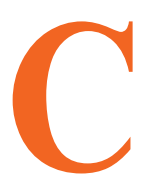

ancer is among the main causes of death and disability, globally (Hekmatpou \& Mehrabi, 2018). Increased prevalence of cancer in recent years and its multiple effects on the biopsychosocial and other life dimensions of the sufferers, has made cancer the main problem of the century (Hekmatpou \& Mehrabi 2018; Pourkiani et al. 2010). Leukemia is classified as cancer. According to the latest World Health Organization (WHO) statistics, the incidence of leukemia is 437000 worldwide. Furthermore, its death rate is 309000 per year, and 217000 people will be added to this population by 2040 (IARC, World Cancer Report 2018).

Leukemia is the seventh most common cancer after breast, stomach, colorectal, lung, bladder, and prostate cancers in Iran. In addition, the number of people affected by this disease is higher than the global statistics and accounts for $5.65 \%$ of all newly diagnosed cancers. Leukemia is also the reason for $7.44 \%$ of all cancer deaths in Iran (IARC, World Cancer Report2018; Musarezaie et al. 2014). In leukemia, the malignant cells originate from the hematopoietic system, and its increase in bone marrow shows itself with or without the involvement of peripheral blood cells (Holzner et al. 2004). The disease consists of two large groups of myeloid and lymphoid, each of them can be acute or chronic, and fall into the 4 categories, including Acute Myeloid Leukemia (AML), Acute Lymphocytic Leu- kemia (ALL), Chronic Myeloid Leukemia (CML), and Chronic Lymphocytic Leukemia (CLL).

The goal of treating hematological malignancies is to increase patients' survival, improve their ability, and maintain an appropriate Quality of Life (QoL) level (Montgomery et al. 2002). The treatment of these malignancies is not generally feasible, and the purpose of treatment is to eliminate symptoms and improve survival. Therefore, QoL has been considered as the main criterion for evaluating patients' responses to the treatment (Pamuk et al. 2008; Madmoli et al. 2017). Patients with leukemia receive different interventions, like chemotherapy, which significantly affects their QoL.

In other words, those receiving chemotherapies have lower QoL scores, and their anxiety rate is higher than the others (Habek et al. 2004; Redaelli et al. 2004). This disease severely changes patient's lifestyle (Santos et al. 2006); therefore, the patients' efforts and their family cooperation are required for optimal adaptation (Golchin et al. 2008). Consequently, QoL has been a spotlight on healthcare area, especially for more than a decade in chronic diseases (Chagani et al. 2017). The interest in evaluating and improving the QoL of patients with chronic diseases, like cancer and their daily functions improvement has significantly enhanced, recently (Safaee et al. 2010).

Patients' survival is not the only concern; because the basis of nursing, philosophy is improving the life expec- 
tancy of those in need of a better life. Nurses' long and direct contact with patients plays a vital role in evaluating and improving their QoL. Furthermore, studies have suggested that nursing interventions have significantly reduced the treatment side-effects of patients with cancer and improved their QoL (Zeighami Mohammedi et al. 2008). Recent advances in cancer treatment improved patients' life expectancy; therefore, QoL phenomena has become more important (Hekmatpou \& Mehrabi 2018).

Some studies have been conducted on the QoL of patients with leukemia in Iran; however, data are scarce in this regard, because these studies disregarded its different types. Information about the QoL of patients with different leukemia types not only paves the way for influential therapies but also leads to more effective interventions. Furthermore, by identifying different aspects of these patients' QoL, those who need more attention will be identified for receiving a comprehensive care plan. Accordingly, the present study aimed to compare the QoL of hospitalized patients with all types of leukemia.

\section{Materials and Methods}

This was a descriptive cross-sectional study. One hundred patients with leukemia hospitalized in Ayatollah Khansari Hospital in Arak City, Iran were selected by purposive sampling method. Moreover, they were assessed for 6 months in 2018. The inclusion criteria were admission to the oncology and hematology ward with at least 3 months passed since cancer diagnosis. Exclusion criteria included transferring to other wards (e.g. ICU), patients or their immediate family death, and cancer metastasis. A cancer diagnosis was based on the laboratory and imaging tests, confirmed by an oncologist. The researcher, while expressing the research objectives, obtained the patients' written consents. Necessary explanations, such as optional participation in the study, the confidentiality of the provided information and possibility of withdrawal from the study were provided to selected patients.

The data gathering tools were a demographic form and the QoL questionnaire for cancer patients approved by the European Organization for Research and Treatment of Cancer (EORTC). Demographic questions included patients' age, gender, marital status, the number of children, occupation, academic background, residence type, insurance status, cancer type, illness duration, as well as the history of past specific diseases and its related treatments.
EORTC QLQ-C30 QoL questionnaire is a standard scale belonging to EORTC and used to assess cancer patients' QoL (Safaee et al. 2007). The questionnaire has 30 questions in 5 functional subscales, including physical condition (five questions), role play (two questions), emotional function (two questions), cognitive function (four questions), and social function (two questions), questions referred to symptoms (fatigue, nausea, vomiting, pain, shortness of breath, sleep disturbances, appetite loss, constipation, diarrhea, financial problems), and overall QoL. To determine QoL, the analyzed data were classified into three levels; good (scores: $>75$ ), relatively good (scores: 50-75), and poor (scores: <50). Higher scores in functional subscale and overall QoL indicate a better patient status in that area; higher scores related to symptoms indicate a lower QoL.

The face validity of the Persian version of the QLQ-C30 has been confirmed, and its Cronbach's alpha reliability was between 0.76 and 0.93 ; thus, it can be used in epidemiological and clinical cancer studies with confidence. The third version of this questionnaire has been validated in Iran (Safaee et al. 2007, 2008a) and its Persian version is also confirmed by EORTC (Safaee et al. 2008a; Safaee et al. 2008b). Descriptive statistics, including frequency, mean, and standard deviation, were used. Statistical tests such as Chi-squared test, Fisher's exact test, and t-test were also applied. Acquired data were analyzed by SPSS V. 21. $\alpha \leq 0.5$ was considered as significant.

\section{Results}

Among the patients, 53\% were males, and $47 \%$ were females with a Mean \pm SD age of $51 \pm 83.16$ years. Regarding leukemia type, $40 \%$ were diagnosed with chronic lymphocytic leukemia, 10\% with acute lymphocytic leukemia, 24\% with chronic myeloid leukemia, and $26 \%$ were admitted with acute myeloid leukemia (Table 1). Based on the findings, the overall Mean $\pm \mathrm{SD}$ QoL score was $62.2 \pm 12.59$, which was at a relatively good range. The Mean \pm SD QoL scores for different leukemia types were $60.10 \pm 14.31$ for chronic lymphocytic leukemia, $59.51 \pm 12.83$ for acute lymphocytic leukemia, $66.61 \pm 10.94$ for chronic myeloid leukemia, and $62.84 \pm 6.33$ for acute myeloid leukemia.

Among the different subscales of QoL, patients with ALL received the lowest score, while patients with CML acquired the highest score. In the emotional dimension, patients with AML gained the lowest score and those with CML had the highest score. In cognitive dimension, patients with ALL had the lowest score and those with AML obtained the highest score. Finally, in social 
Table 1. Frequency distribution of patients with leukemia based on demographic variables

\begin{tabular}{ccc}
\hline Status & Variable & No. (\%) \\
\hline Gender & Female & $53(53 \%)$ \\
& Male & $47(47 \%)$ \\
\hline Marrital Status & Single & $47(47 \%)$ \\
& Married & $40(40 \%)$ \\
\hline Age & Other & $13(13 \%)$ \\
\hline Mean $\pm S D$ & $51.83 \pm 16$ \\
\hline Leukemia type & AML & 26 (26\%) \\
& ALL & $10(10 \%)$ \\
& $\mathrm{CML}$ & $24(24 \%)$ \\
& $\mathrm{CLL}$ & 40 (40\%) \\
\hline
\end{tabular}

Table 2. Comparing Mean \pm SD scores of total QoL and its subscales in patients with different leukemia types

\begin{tabular}{|c|c|c|c|c|c|}
\hline \multirow{2}{*}{ Variable } & \multicolumn{4}{|c|}{ Mean \pm SD } & \multirow{2}{*}{$\mathbf{P}$} \\
\hline & AML & CML & ALL & CLL & \\
\hline Physical function & $11.38 \pm 7.87$ & $12.08 \pm 2.7$ & $8.40 \pm 2.90$ & $12.05 \pm 7.22$ & 0.03 \\
\hline Role function & $3.53 \pm 1.63$ & $3.58 \pm 2.01$ & $2.40 \pm 0.83$ & $3.15 \pm 1.61$ & 0.025 \\
\hline Emotional function & $7.7 \pm 3.08$ & $9.33 \pm 2.83$ & $9.00 \pm 1.24$ & $7.70 \pm 6.21$ & 0.04 \\
\hline Cognitive function & $3.96 \pm 1.08$ & $3.25 \pm 1.7$ & $8.20 \pm 0.63$ & $3.42 \pm 1.1$ & 0.02 \\
\hline Social function & $2.57 \pm 0.808$ & $3.91 \pm 1.95$ & $2.40 \pm 2.44$ & $2.85 \pm 1.16$ & 0.01 \\
\hline Global health status & $9.42 \pm 2.56$ & $7.79 \pm 3.17$ & $7.10 \pm 3.16$ & $7.50 \pm 3.01$ & 0.02 \\
\hline Fatigue & $6.03 \pm 1.28$ & $7.70 \pm 1.89$ & $7.40 \pm 1.05$ & $5.60 \pm 2.11$ & 0.00 \\
\hline Nausea and vomiting & $3.03 \pm 1.24$ & $3.91 \pm 2.33$ & $2.20 \pm 0.42$ & $2.95 \pm 1.37$ & 0.01 \\
\hline Pain & $4.57 \pm 2.94$ & $3.83 \pm 1.99$ & $5.30 \pm 1.64$ & $55.3 \pm 1.63$ & 0.02 \\
\hline Dyspnea & $1.53 \pm 0.64$ & $1.58 \pm 0.97$ & $2.10 \pm 0.42$ & $1.35 \pm 0.73$ & 0.06 \\
\hline Insomnia & $2.11 \pm 0.76$ & $2.00 \pm 1.28$ & $2.20 \pm 1.22$ & $9.10 \pm 0.98$ & 0.04 \\
\hline Appetite loss & $1.88 \pm 0.65$ & $7.10 \pm 1.04$ & $3.10 \pm 0.48$ & $9.10 \pm 1.07$ & 0.02 \\
\hline Constipation & $1.88 \pm 0.71$ & $1.58 \pm 0.65$ & $4.20 \pm 1.07$ & $62.1 \pm 0.74$ & 0.00 \\
\hline Diarrhea & $1.38 \pm 0.69$ & $1.25 \pm 0.44$ & $8.10 \pm 0.78$ & $50.1 \pm 0.50$ & 0.02 \\
\hline Financial difficulties & $2.08 \pm 0.87$ & $2.62 \pm 0.96$ & $6.20 \pm 0.51$ & $2.07 \pm 0.91$ & 0.05 \\
\hline Total QoL & $62.84 \pm 10.63$ & $66.16 \pm 10.94$ & $59.41 \pm 12.83$ & $60.1 \pm 14.31$ & 0.03 \\
\hline
\end{tabular}


dimension, patients with AML and those with ALL had the lowest and highest scores, respectively (Table 2).

Regarding the experienced symptoms, fatigue was the most annoying symptom reported by the samples. Among the symptoms, nausea and vomiting were higher in CML, pain in AML, dyspnea in CML, decreased impaired olfactory sense in ALL, reduced appetite in CLL, and diarrhea and constipation in patients with ALL (Table 2). Female gender, having a university degree, being a housewife, and living in rural areas were associated with higher QoL score. There was a significant relationship between age, residency, and marital status, and total QoL $(\mathrm{P}<0.05)$.

\section{Discussion}

QoL is regarded as a sign of responding to treatment and is a part of a disease control program. By analyzing the data related to this concept, a valuable source of information will be available to evaluate the quality of healthcare services and preventive interventions.

This study compared the QoL of hospitalized patients with all types of leukemia. It also assessed the relationship between QoL and patients' characteristics. The patients' overall Mean \pm SD QoL score was 62.29 \pm 12.59 , which was at a relatively good level. This finding was consistent with some other studies (Dehkordi et al. 2006; Shaban et al. 2004). Furthermore, compared to prior studies, the patients' QoL was better; this could be due to the differences in biopsychological support and care provided by patients' families (Glaus et al. 2000; Lee et al. 2001; Sammarco et al. 2001). In terms of QoL in patients with all types of leukemia, those with CML had the highest QoL score $16.69 \pm 10.94$. In addition, the patients with ALL had a lower QoL; therefore, those with ALL require more attention.

Our patients had experienced many physical symptoms due to the disease process and received treatments. Generally, patients with hematologic malignancies have significant physical symptoms, such as fatigue, insomnia, drowsiness, pain, shortness of breath and neuropathy, diarrhea, vomiting, and olfactory disorder, as a complication of cancer progression and their treatment side effects (Buckley et al. 2017; Verleden et al. 2010). Moreover, about $63 \%$ of patients with leukemia, experience at least one treatment side effect (Fulbright et al. 2011; Kopp et al. 2012).

The Mean \pm SD age of studied patients was $51.83 \pm 16$ years. In Pakistan, the mean age of patients in a related study was 48 years, which is close to ours (Chagani et al. 2017); however, according to Cella et al. (2012), the average age of leukemia patients was 60.1 years. It seems that this 10-year difference in the average age of the disease onset between the current study and Cella et al. study in the United States could be related to the higher level of QoL in the US, which delays cancer occurrence (Cella et al. 2012).

Assessing different QoL dimensions in our study suggested that the weakest performance is in the social area; this is consistent with some other studies in Iran (Esmailnasab Taymoori \& Darabi 2013; Mardani Hamule et al. 2009). Furthermore, the best performance belonged to the physical dimension which is not consistent with the results of Esmailnasab et al. (2013), Blazeby et al. (2005), and Kobayashi et al. (2008); they found that cognitive function obtained the highest score (Esmailnasab Taymoori \& Darabi 2013; Blazeby et al. 2005; Kobayashi et al. 2008).

QoL reflects the biopsychosocial functions of individuals in response to the diseases and received treatments (Mohaddesi et al. 2013). In our study, patients with ALL received the lowest QoL score, while those with CML acquired the highest. Among the different dimensions of QoL, patients with AML gained the lowest score in the emotional dimension, and those with CML had the highest one. In cognitive dimension, patients with ALL had the lowest score and patients with AML obtained the highest; whereas, in another study, patients with CML obtained the lowest score in emotional dimension (Shanafelt et al. 2007). However, in another study, the type of leukemia was not significantly associated with patients' QoL (Hadi, Namvar \& Montazeri 2011). This difference may be because, in all four types of leukemia, most patients received chemotherapy, they were often hospitalized, and involved with the same infection and disease complications (Hadi, Namvar \& Montazeri 2011). Similar results were observed in a study by Santos et al. (2006).

Generally, the findings revealed that patients with myeloid leukemia had a better QoL, compared to those with lymphocytic leukemia. In addition, the needs of patients with leukemia are different according to leukemia type. Accordingly, the knowledge of nurses and other healthcare staff about these findings could promote care and help to improve the QoL of patients' with different leukemia types. 


\section{Ethical Considerations}

\section{Compliance with ethical guidelines}

This study was approved in the Medical Ethics Committee of Arak University of Medical Sciences (Code: IR.ARAKMU.REC.1393.175.18). The study participants were assured of the confidentiality of their information and provided a written consent form to participate in the study.

\section{Funding}

This project was supported by the Vice-Chancellor of Research Department of Arak University of Medical Sciences.

\section{Authors' contributions}

All authors contributed to this article and read and approved the final manuscript for submission.

\section{Conflict of interest}

There are no conflicts of interest to be declared by the authors.

\section{Acknowledgments}

The authors gratefully acknowledge the Research Council of Arak University of Medical Sciences for its financial support.

\section{References}

Blazeby, J. M., et al., 2005. A prospective comparison of quality of life measures for patients with esophageal cancer. Quality of Life Research, 14(2), pp. 387-93. [DOI:10.1007/s11136-004-06224] [PMID]

Buckley, S. A., et al., 2017. Determinants of quality of life in patients with acute myeloid leukemia. Journal of Clinical Oncology, 30(7), pp. 23-34.

Cella, D., et al., 2012. Measuring health-related quality of life in leukemia: The Functional Assessment of Cancer TherapyLeukemia questionnaire (FACT-Leu). Value in Health, 15(8), pp. 1051-8.

Chagani, P., et al., 2017. Quality of life and its determinants in adult cancer patients undergoing chemotherapy treatment in Pakistan. Asia-Pacific Journal of Oncology Nursing, 4(2), pp. 1406. [DOI:10.4103/2347-5625.204499] [PMID] [PMCID]
Dehkordi, B., et al., 2006. [The study of validity and reliability of the quality of life questionnaire in patients with cancer (Persian)]. Journal of Armaghan-e Danesh, 12, pp.79-87.

Esmailnasab, N., Taymoori, P. \& Darabi, F., 2013. [Comparison of quality of life 3 and 9 months after surgery in patients with gastrointestinal cancer (Persian)]. The Journal of Urmia University of Medical Sciences, 24(4), pp. 226-34.

Fulbright, J. M., et al., 2011. Late effects of childhood leukemia therapy. Current Hematologic Malignancy Reports, 6(3), pp. 195205. [DOI:10.1007/s11899-011-0094-x] [PMID]

Glaus, A., \& Müller, S., 2000. [Hemoglobin and fatigue in cancer patients: inseparable twins? (German)] Schweizerische Medizinische Wochenschrift, 130(13), pp. 471-7. [PMID]

Golchin, M. E., et al., 2008. Effects of self care program on quality of life in patients with acute leukemia receiving chemotherapy. Zahedan Journal of Research in Medical Sciences, 10(3), pp. 2-11. [PMID] [PMCID]

Habek, D., et al., 2004. Acute abdomen as first symptom of acute leukemia. Archives of Gynecology and Obstetrics, 270(2), pp. 122-3. [DOI:10.1007/s00404-002-0453-x] [PMID]

Hadi, N., Namvar, E., \& Montazeri, A., 2011. [Health-related quality of life in adults lukemic patients (Persian)]. Payesh, 10(2), pp. 151-6.

Hekmatpou, D. \& Mehrabi, F., 2018. Exploratory study on diagnosed cancers and quality of life of hospitalized patients. Journal of Nursing and Midwifery Sciences, 5(3), pp. 109-15. [DOI:10.4103/JNMS.JNMS_15_18]

Holzner, B., et al., 2004. Quality of life of patients with chronic lymphocytic leukemia: Results of a longitudinal investigation over 1 yr. European Journal of Haematology, 72(6), pp. 381-9. [DOI:10.1111/j.1600-0609.2004.00233.x] [PMID]

Kobayashi, K., et al., 2008. Effects of socioeconomic factors and cancer survivors' worries on their Quality of Life (QOL) in Japan. Psycho-Oncology, 17(6), pp. 606-11. [DOI:10.1002/ pon.1278] [PMID]

Kopp, L. M., et al., 2012. Late effects in adult survivors of pediatric cancer: A guide for the primary care physician. The American Journal of Medicine, 125(7), pp. 636-41. [DOI:10.1016/j.amjmed.2012.01.013] [PMID]

Lee, E. H., 2001. Fatigue and hope: Relationships to psychosocial adjustment in Korean women with breast cancer Applied Nursing Research, 14(2), pp. 87-93. [DOI:10.1053/ apnr.2001.22374] [PMID]

Madmoli, M. et al., 2017. Quality of life and some related factors in patients with beta thalassemia major in Southwest Iran. Journal of Client-Centered Nursing Care, 3(2), pp. 139-46. [DOI:10.32598/jccnc.3.2.139]

Mardani, H. M, Shahraki, V.A., 2009. [The assessment of relationship between mental health and quality of life in cancer patients (Persian)]. Scientific Journal of Hamadan University of Medical Sciences, 16(2), pp.33-8.

Mohaddesi, H., et al., 2013. [Quality of life in breast cancer women undertreating in cancer reaserch center (Persian)]. Iranian Quarterly Journal of Breast Disease, 5(4), pp. 35-43. 
Montgomery, C., et al., 2002. Individual quality of life in patients with Leukaemia and Lymphoma. Psycho-Oncology, 11(3), pp. 239-43. [DOI:10.1002/pon.557] [PMID]

Musarezaie, A., et al., Factors affecting quality of life and fatigue in patients with leukemia under chemotherapy. Journal of Education and Health Promotion, 3(1), pp. 64-72. [PMID] [PMCID]

Pamuk, G. E., et al., 2008. EORTC QLQ-C30 assessment in Turkish patients with hematological malignancies. Annals of Hematology, 87(4), pp. 305-310. [DOI:10.1007/s00277-008-0445-4] [PMID]

Pourkiani, M., et al., 2010. [Does a rehabilitation program improve quality of life in breast cancer patients? (Persian)] Payesh, 9(2), pp. 61-8.

Redaelli, A., et al., 2004. Short-and long-term effects of acute myeloid leukemia on patient health-related quality of life. Cancer Treatment Reviews, 30(1), pp. 103-17. [DOI:10.1016/S03057372(03)00142-7]

Safaee, A. \& Moghim Dehkordi, B., 2007. Validation study of a Quality of Life questionnaire (QOL) for use in Iran. Asian Pacific Journal of Cancer Prevention, 8(4), pp. 543-6. [PMID]

Safaee, A., et al., 2008a. Predictors of quality of life in breast cancer patients under chemotherapy. Indian Journal of Cancer, 45(3), pp. 107-11. [DOI:10.4103/0019-509X.44066] [PMID]

Safaee, A., et al., 2008b. Quality of life and related factors in breast cancer patients under chemotherapy. Iranian Journal of Epidemiology, 3(3), pp. 61-6. [DOI:10.4103/0019-509X.44066] [PMID]

Safaee, A., et al., 2010. [Cancer-related fatigue in breast cancer patients under chemotherapy (Persian)]. Koomesh, 11(4), pp. $317-22$.

Sammarco, A., 2001. Perceived social support, uncertainty, and quality of life of younger breast cancer survivors. Cancer Nursing, 24(3), pp. 212-9. [DOI:10.1097/00002820-200106000-00007] [PMID]

Santos, F. R., et al., 2006. Psychosocial adaptation and quality of life among Brazilian patients with different hematological malignancies. Journal of Psychosomatic Research, 60(5), pp. 505-11. [DOI:10.1016/j.jpsychores.2005.08.017] [PMID]

Shaban, M., et al., 2004. [The relation between the cancer characteristics and quality of life in the patients under chemotherapy (Persian)]. Hayat, 10(3), pp.79-84.

Shanafelt, T. D., et al., 2007. Quality of life in chronic lymphocytic leukemia: An international survey of 1482 patients. British Journal of Haematology, 139(2), pp. 255-64. [DOI:10.1111/j.13652141.2007.06791.x] [PMID]

Verleden, S., Noens, L., \& Audenaert, K., 2010. Quality of life in adult de novo acute leukemia patients. Blood Journal, 116(21), pp. $47-57$.

World Health Organization. World Cancer Report 2018. Geneva: World Health Organization.

Zeighami Mohammedi, Sh., Hushmand, P., \& Kooshyar, M. M., 2008. Quality of life in cancer patients undergoing chemotherapy. Journal of Hamadan Nursing and Midwifery Faculty, 16(1), pp. 5-15. 
This Page Intentionally Left Blank 